hep-ph/0402215

\title{
Electroweak Symmetry Breaking via UV Insensitive Anomaly Mediation
}

\author{
Ryuichiro Kitano, Graham D. Kribs, and Hitoshi Murayama* \\ School of Natural Sciences, Institute for Advanced Study, Princeton, NJ 08540 \\ kitano@ias.edu, kribs@ias.edu, murayama@ias.edu
}

\begin{abstract}
Anomaly mediation solves the supersymmetric flavor and CP problems. This is because the superconformal anomaly dictates that supersymmetry breaking is transmitted through nearly flavor-blind infrared physics that is highly predictive and UV insensitive. Slepton mass squareds, however, are predicted to be negative. This can be solved by adding $D$-terms for $\mathrm{U}(1)_{Y}$ and $\mathrm{U}(1)_{B-L}$ while retaining the UV insensitivity. In this paper we consider electroweak symmetry breaking via UV insensitive anomaly mediation in several models. For the MSSM we find a stable vacuum when $\tan \beta<1$, but in this region the top Yukawa coupling blows up only slightly above the supersymmetry breaking scale. For the NMSSM, we find a stable electroweak breaking vacuum but with a chargino that is too light. Replacing the cubic singlet term in the NMSSM superpotential with a term linear in the singlet we find a stable vacuum and viable spectrum. Most of the parameter region with correct vacua requires a large superpotential coupling, precisely what is expected in the "Fat Higgs" model in which the superpotential is generated dynamically. We have therefore found the first viable UV complete, UV insensitive supersymmetry breaking model that solves the flavor and CP problems automatically: the Fat Higgs model with UV insensitive anomaly mediation. Moreover, the cosmological gravitino problem is naturally solved, opening up the possibility of realistic thermal leptogenesis.
\end{abstract}

\footnotetext{
*On leave of absence from Department of Physics, University of California, Berkeley, CA 94720.
} 


\section{Introduction}

The electroweak scale of the Standard Model is destabilized by quadratically divergent radiative corrections to the Higgs mass squared. It has long been known that supersymmetry provides an elegant resolution to this naturalness problem by providing superpartners that cancel the quadratic divergences to all orders in perturbation theory. The absence of experimental evidence for superpartners roughly requires that they acquire supersymmetry breaking masses larger than of order the electroweak scale. The cancellation of the quadratic divergence is therefore inexact, requiring a fine-tuning of about one part in $\left(m_{\mathrm{SUSY}} / M_{Z}\right)^{2}$. This fine-tuning is minimized when the size of soft supersymmetry breaking is as close as possible to $M_{Z}$ [1].

The generic difficulty with such low superpartner masses is that they can lead to large contributions to flavor changing neutral currents (FCNC) and $\mathrm{CP}$ violating interactions at low energy. Flavor-generic supersymmetry breaking leads to effective FCNC and CP violating operators that must be suppressed by tens to hundreds of $\mathrm{TeV}$ to avoid conflict with experiment. Raising the supersymmetry breaking scale sufficiently does solve the supersymmetric flavor problem, but at the expense of reintroducing a fine-tuning of at least one part in $10^{6}$. Avoiding this fine-tuning problem requires carefully arranging supersymmetry breaking to be mediated to the minimal supersymmetric standard model (MSSM) in a flavor-blind way. ${ }^{\dagger}$

There are only a few known flavor-blind mediation mechanisms: gauge mediation [2], anomaly

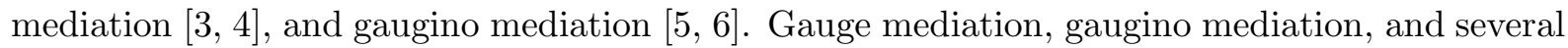
viable versions of anomaly mediation [0, 8, 9, 10, 11, 12, 13, 14, 15, 16] rely on specific UV physics to ensure flavor-blindness and an acceptable spectrum. Gauge mediation generically requires a messenger sector to which the MSSM is coupled only through gauge fields. Gaugino mediation requires an extra dimension (or deconstruction [17, 18]) with only gauge fields propagating in the bulk. Both frameworks require that the physics that explains the origin of flavor, such as Froggatt-Nielsen mechanism [19], must be well above the mediation scale such that the supersymmetric flavor problem is not reintroduced. Anomaly mediation requires a mechanism to suppress supergravity contributions (sequestering across a bulk [3] or from a CFT [20, 21]) as well as a mechanism to generate positive slepton mass squareds. A qualitatively different approach, UV insensitive anomaly mediation [11, 22] is the idea that soft breaking depends only on infrared (IR) physics through anomaly mediation (sequestered using a bulk [22] or CFT [23]) and $D$-terms for hypercharge and $B-L$ (see also [24, 25]).

UV insensitive supersymmetry breaking is highly predictive since all the supersymmetry breaking terms except for the $D$-terms can be calculated with known low energy coupling constants. The UV implementation of this model requires sequestering, to prevent the usual supergravity contributions to scalar masses, as well as a mechanism to generate $D$-terms [11, 22, 23]. The virtue of this approach, however, is that the soft breaking masses are independent of these details of the UV physics. In this paper we carefully examine electroweak symmetry breaking in the MSSM and extensions with a singlet superfield with supersymmetry breaking communicated via UV insensitive anomaly mediation.

\footnotetext{
${ }^{\dagger}$ An alternative possibility is to constrain the form of soft supersymmetry breaking parameters using flavor symmetries. However, the CP violation is difficult to forbid unless the CP symmetry is exact at high energies and is broken only spontaneously.
} 
This paper is organized as follows. In Sec. 2 we review anomaly mediation and the UV insensitive model with $D$-terms. In Sec. 3 we consider UV insensitive anomaly mediation with the MSSM particle content. We find that a minimum of the potential is obtained only when the top Yukawa coupling is large, of order three, and the ratio of Higgs vacuum expectation values $\left(\tan \beta=\left\langle H_{2}\right\rangle /\left\langle H_{1}\right\rangle\right)$ is much less than 1. The large top Yukawa coupling runs into a Landau pole immediately above the supersymmetry breaking scale. The MSSM with UV insensitive anomaly mediation is therefore UV incomplete and must be replaced with an unknown theory above that scale. This does not cause an inconsistency of our framework because the soft terms are, by definition, UV insensitive. However, the large top Yukawa coupling prevents us from obtaining an accurate estimate of the mass of the lightest Higgs boson due to the importance of higher order radiative corrections.

We then consider extensions of the MSSM particle content by adding a singlet superfield in the hopes of finding a UV complete and calculable model. In Sec. 4 we first consider the nextto-minimal supersymmetric standard model (NMSSM) with the superpotential $W=\lambda S H_{1} H_{2}+$ $(h / 3) S^{3}$, where $S$ is the singlet superfield and $H_{1}$ and $H_{2}$ are the doublet Higgs superfields. We find that this model is not viable because the chargino mass is predicted to be much too small. Then in Sec. 5 we consider an NMSSM-like model with superpotential $W=\lambda S H_{1} H_{2}+m^{2} S$ and $\lambda \gtrsim 1$. The superpotential coupling $\lambda$ runs into a Landau pole at an intermediate scale, implying that this model requires a UV completion just like the MSSM. Fortunately we know precisely what the UV completion of this superpotential is: the "Fat Higgs" model [26]! The Fat Higgs model is a theory with $\mathrm{SU}(2)$ gauge group and three (or four) flavors that gets strong at an intermediate scale. These flavors confine into composite mesons with a dynamically generated superpotential that is precisely of the form given above. What is perhaps most fascinating is that we find the parameter region with a stable electroweak breaking vacuum occurs for values of the couplings that are quite ordinary from the viewpoint of the Fat Higgs model. Furthermore, the supersymmetric CP problem is solved automatically, and the traditional $\mu$-problem is solved. (A new $\mu$-like problem resurfaces in the three-flavor Fat Higgs model, but unlike other flavorblind models, the solution to this new $\mu$-problem does not reintroduce the supersymmetric CP problem.) Thus, the Fat Higgs model is the first calculable UV complete model with UV insensitive anomaly mediation that automatically solves both the supersymmetric flavor and CP problems.

\section{Anomaly Mediation}

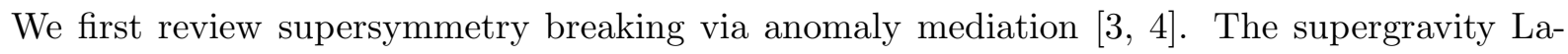
grangian can be obtained from a local superconformal theory by a gauge fixing of extra symmetries 27, 28, 29]. The gauge fixing can be done by setting the values of the components of a compensator chiral multiplet $\Phi$. By construction, $\Phi$ couples to the violation of conformal symmetry, i.e., any dimensionful parameters such as mass parameters and also the renormalization scale. The scalar component of $\Phi$ is determined such that the gravity kinetic term has the canonical normalization. Supersymmetry breaking in the hidden sector causes the auxiliary component $F_{\Phi}$ to acquire a non-vanishing value to cancel the cosmological constant. Since the vacuum energy from supersymmetry breaking is its own order parameter, $F_{\Phi}$ is proportional to 
the gravitino mass $m_{3 / 2}$. With an appropriate redefinition of $\Phi$, the scalar component can be fixed to be 1 and $F_{\Phi}=m_{3 / 2}$ in this normalization.

Anomaly mediation is the contribution to the soft terms originated from $F_{\Phi}$. The classical Lagrangian is given by

$$
\begin{aligned}
\mathcal{L}= & \int d^{4} \theta\left[Q_{i}^{\dagger} e^{-2 V} Q_{i}\right]+\left(\int d^{2} \theta \frac{1}{2 g^{2}} \operatorname{Tr}\left[W^{\alpha} W_{\alpha}\right]+\text { h.c. }\right) \\
& -\left(\int d^{2} \theta\left[\lambda_{i j k} Q_{i} Q_{j} Q_{k}+\Phi m_{i j} Q_{i} Q_{j}+\Phi^{2} v_{i}^{2} Q_{i}\right]+\text { h.c. }\right),
\end{aligned}
$$

where $Q_{i}$ and $V$ are the chiral and vector superfields, and $W^{\alpha}$ is the field strength made of $V$. We can read off the tree-level soft terms by substituting $\Phi=1+m_{3 / 2} \theta^{2}$ into the above Lagrangian, resulting in

$$
\mathcal{L}_{\text {soft }}=\left(-B m_{i j} q_{i} q_{j}-C v_{i}^{2} q_{i}+\text { h.c. }\right)
$$

where $B=m_{3 / 2}, C=2 m_{3 / 2}$, and $q_{i}$ is the scalar component of the chiral superfield $Q_{i}$. Since the compensator also couples to the renormalization scale, $\mu$, there are additional contributions at quantum level. The $\mu$ dependence appears in the wave function renormalization and the gauge coupling as follows:

$$
\begin{aligned}
\mathcal{L}= & \int d^{4} \theta\left[Z_{i}\left(\frac{\mu}{\sqrt{\Phi \Phi^{\dagger}}}\right) Q_{i}^{\dagger} e^{-2 V} Q_{i}\right]+\left(\int d^{2} \theta \frac{1}{2 g^{2}\left(\frac{\mu}{\Phi}\right)} \operatorname{Tr}\left[W^{\alpha} W_{\alpha}\right]+\text { h.c. }\right) \\
& -\left(\int d^{2} \theta\left[\lambda_{i j k} Q_{i} Q_{j} Q_{k}+\Phi m_{i j} Q_{i} Q_{j}+\Phi^{2} v_{i}^{2} Q_{i}\right]+\text { h.c. }\right)
\end{aligned}
$$

Expanding $Z_{i}$ and $g^{2}$ in terms of $\theta^{2}$ and redefining the fields to have canonical kinetic terms, we obtain trilinear scalar couplings $\left(A_{i j k}\right)$, scalar mass squareds $\left(\tilde{m}^{2}\right)$, and a gaugino mass $\left(m_{\lambda}\right)$ as follows ${ }^{\ddagger}$ :

$$
A_{i j k}=-\lambda_{i j k}\left(\gamma_{i}+\gamma_{j}+\gamma_{k}\right) m_{3 / 2}, \quad \tilde{m}_{i}^{2}=\frac{1}{2} \dot{\gamma}_{i} m_{3 / 2}^{2}, \quad m_{\lambda}=\frac{\beta}{g} m_{3 / 2},
$$

where the anomalous dimension $\gamma_{i}$ and the beta function $\beta$ are defined by

$$
\gamma_{i}=-\frac{1}{2} \frac{d}{d t} \log Z_{i}(\mu), \quad \dot{\gamma}_{i}=\frac{d}{d t} \gamma_{i}, \quad \beta=\frac{d}{d t} g, \quad t=\log \mu .
$$

The soft parameters above are defined by the Lagrangian

$$
\mathcal{L}_{\text {soft }}=-\left(A_{i j k} q_{i} q_{j} q_{k}+\text { h.c. }\right)-\tilde{m}_{i}^{2}\left|q_{i}\right|^{2}-\frac{1}{2} m_{\lambda} \bar{\lambda} \lambda .
$$

The results in Eq. (2.4) are true at any energy scale since the form of the Lagrangian (2.3) is always valid. This indicates that the soft terms at a low-energy scale depend only on the anomalous dimensions or beta functions at that scale and do not care about the theory at higher energies. This UV insensitivity is the main feature of the anomaly mediation and implies that

\footnotetext{
${ }^{\ddagger}$ The gaugino mass parameter $m_{\lambda}$ flips its sign when we redefine the gauginos $\lambda \rightarrow i \lambda$ in order to get rid of the factor of $i$ in front of the gaugino-scalar-fermion vertex.
} 
the soft terms are calculable and nearly flavor-blind since the gauge contribution overwhelms the Yukawa coupling contribution for all but the top squarks.

The problem is that slepton mass squareds are predicted to be negative. This can be easily seen by an explicit calculation with the formula in Eq. (2.4). Neglecting the Yukawa interactions of the leptons, the slepton masses are given by

$$
m_{\tilde{l}}^{2}=\left(-\frac{1}{2} b_{Y} g_{Y}^{4}-\frac{3}{2} b_{2} g_{2}^{4}\right) M^{2}, \quad m_{\tilde{e}^{c}}^{2}=-2 b_{Y} g_{Y}^{4} M^{2}
$$

where $M=m_{3 / 2} /(4 \pi)^{2}$. The slepton masses depend overwhelmingly on the breaking of conformal symmetry by the gauge beta functions that are given by

$$
\beta_{a}=\frac{b_{a} g_{a}^{3}}{16 \pi^{2}}
$$

where the beta function coefficients $\left[b_{Y}, b_{2}, b_{3}\right]$ are $[11,1,-3]$ for $\left[\mathrm{U}(1)_{Y}, \mathrm{SU}(2)_{L}, \mathrm{SU}(3)_{c}\right]$ gauge symmetries. Upon inserting these numbers into the above expressions for the slepton masses one immediately sees that the slepton mass squareds are negative, and therefore "pure" anomaly mediation is excluded.

For anomaly mediation to be viable there must be an additional contribution to the slepton masses. Additional contributions generically involve UV sensitive physics [0, 8, 9, 10, 11, 12, 13, 14, 15, 16], that can lead to a perfectly viable supersymmetry breaking model. However, the anomaly-mediated soft mass predictions, including UV insensitivity, are generally lost. An interesting alternative possibility is to add $D$-term contributions to the sfermion masses [11] that have been shown to preserve UV insensitivity [22]. If some non-MSSM fields charged under a U(1) gauge symmetry acquire vacuum expectation values (VEVs) at a slightly deviated point from the $D$-flat direction due to a supersymmetry breaking effect, the $D$-term generates an additional contribution to sfermion masses proportional to the $\mathrm{U}(1)$ charges through the coupling of $-g \mathcal{Q}_{i} D\left|q_{i}\right|^{2}$ in the Lagrangian, where $g$ and $\mathcal{Q}_{i}$ are the gauge coupling constant and the charge of the $q_{i}$ field, respectively. The UV insensitivity was shown to be preserved when the $\mathrm{U}(1)$ symmetry is anomaly free with respect to the standard model gauge group. In the MSSM there are two candidates of the anomaly free $\mathrm{U}(1)$ symmetries, i.e., $\mathrm{U}(1)_{Y}$ and $\mathrm{U}(1)_{B-L}$, and those $D$-term contributions are sufficient to resolve the tachyonic slepton problem as we see below. Although $\mathrm{U}(1)_{Y}$ is unbroken above the electroweak scale, the kinetic mixing between $\mathrm{U}(1)_{B-L}$ and $\mathrm{U}(1)_{Y}$ induces a $D$-term for $\mathrm{U}(1)_{Y}$ [30], once a $D$-term for $\mathrm{U}(1)_{B-L}$ is generated.

The sfermion masses in UV insensitive anomaly mediation can therefore be expressed as a "pure" anomaly mediated piece plus $D$-terms. Evaluating the beta functions and gauge couplings at the weak scale, ${ }^{\S}$ neglecting the Yukawa couplings, we find the first and second generation soft masses to be 22]

$$
\begin{aligned}
& m_{\tilde{l}}^{2}=-0.344 M^{2}+\frac{1}{2} D_{Y}+D_{B-L}, \\
& m_{\tilde{e}^{c}}^{2}=-0.367 M^{2}-D_{Y}-D_{B-L},
\end{aligned}
$$

\footnotetext{
${ }^{\S}$ We evaluated at the scale $\mu=500 \mathrm{GeV}$ for illustration, but threshold corrections should be taken into account for accurate calculations of the physical masses.
} 


$$
\begin{aligned}
m_{\tilde{q}}^{2} & =11.6 M^{2}-\frac{1}{6} D_{Y}-\frac{1}{3} D_{B-L}, \\
m_{\tilde{u}^{c}}^{2} & =11.7 M^{2}+\frac{2}{3} D_{Y}+\frac{1}{3} D_{B-L}, \\
m_{\tilde{d}^{c}}^{2} & =11.8 M^{2}-\frac{1}{3} D_{Y}+\frac{1}{3} D_{B-L},
\end{aligned}
$$

while for the third generation scalars and Higgs bosons

$$
\begin{aligned}
m_{\tilde{l}_{3}}^{2} & =-0.346 M^{2}+\frac{1}{2} D_{Y}+D_{B-L} \\
m_{\tilde{e}_{3}^{c}}^{2} & =-0.371 M^{2}-D_{Y}-D_{B-L}, \\
m_{\tilde{q}_{3}}^{2} & =9.40 M^{2}-\frac{1}{6} D_{Y}-\frac{1}{3} D_{B-L}, \\
m_{\tilde{u}_{3}^{c}}^{2} & =7.37 M^{2}+\frac{2}{3} D_{Y}+\frac{1}{3} D_{B-L}, \\
m_{\tilde{d}_{3}^{c}}^{2} & =11.8 M^{2}-\frac{1}{3} D_{Y}+\frac{1}{3} D_{B-L}, \\
m_{H_{1}}^{2} & =-0.395 M^{2}+\frac{1}{2} D_{Y} \\
m_{H_{2}}^{2} & =-6.79 M^{2}-\frac{1}{2} D_{Y}
\end{aligned}
$$

for $\tan \beta=3$ as an example.

There is a window for the sleptons to be non-tachyonic:

$$
D_{Y}<-D_{B-L}<\frac{1}{2} D_{Y}<0 .
$$

Also, notice that the $D$-term contributions to the left-handed and the right-handed up-type squarks are always negative, so that the $D$-terms cannot be much larger than the anomalymediated contribution to ensure all squark mass squareds stay positive.

For later use we evaluate the soft masses for $\tan \beta=0.3$,

$$
\begin{aligned}
m_{\tilde{l}}^{2} & =-0.344 M^{2}+\frac{1}{2} D_{Y}+D_{B-L}, \\
m_{\tilde{e}^{c}}^{2} & =-0.367 M^{2}-D_{Y}-D_{B-L}, \\
m_{\tilde{q}}^{2} & =11.6 M^{2}-\frac{1}{6} D_{Y}-\frac{1}{3} D_{B-L}, \\
m_{\tilde{u}^{c}}^{2} & =11.7 M^{2}+\frac{2}{3} D_{Y}+\frac{1}{3} D_{B-L}, \\
m_{\tilde{d}^{c}}^{2} & =11.8 M^{2}-\frac{1}{3} D_{Y}+\frac{1}{3} D_{B-L}, \\
m_{\tilde{l}_{3}}^{2} & =-0.344 M^{2}+\frac{1}{2} D_{Y}+D_{B-L}, \\
m_{\tilde{e}_{3}^{c}}^{2} & =-0.368 M^{2}-D_{Y}-D_{B-L}, \\
m_{\tilde{q}_{3}}^{2} & =550 M^{2}-\frac{1}{6} D_{Y}-\frac{1}{3} D_{B-L},
\end{aligned}
$$




$$
\begin{aligned}
m_{\tilde{u}_{3}^{c}}^{2} & =1090 M^{2}+\frac{2}{3} D_{Y}+\frac{1}{3} D_{B-L}, \\
m_{\tilde{d}_{3}^{c}}^{2} & =11.8 M^{2}-\frac{1}{3} D_{Y}+\frac{1}{3} D_{B-L} \\
m_{H_{1}}^{2} & =-0.342 M^{2}+\frac{1}{2} D_{Y} \\
m_{H_{2}}^{2} & =1678 M^{2}-\frac{1}{2} D_{Y} .
\end{aligned}
$$

The large top Yukawa coupling gives a huge enhancement in $m_{\tilde{q}_{3}}^{2}, m_{\tilde{u}_{3}^{c}}^{2}$, and $m_{H_{2}}^{2}$.

\section{Electroweak Symmetry Breaking in the MSSM}

We now discuss electroweak symmetry breaking in the MSSM with UV insensitive ( $D$-term modified) anomaly mediation. We will find a parameter region with the correct electroweak breaking vacuum (the $Z$-boson mass is correct), but $\tan \beta<1$. The top Yukawa coupling is so large in the region that the coupling runs into a Landau pole just above the supersymmetry breaking scale.

The stationary conditions of the potential can be solved analytically at tree-level. The conditions are well known

$$
\begin{aligned}
m_{Z}^{2} & =-\frac{m_{H_{1}}^{2}-m_{H_{2}}^{2}}{\cos 2 \beta}-\left(m_{H_{1}}^{2}+m_{H_{2}}^{2}+2 \mu^{2}\right), \\
\sin 2 \beta & =-\frac{2 B \mu}{m_{H_{1}}^{2}+m_{H_{2}}^{2}+2 \mu^{2}},
\end{aligned}
$$

where $\mu$ is the mass parameter in the superpotential $W=\mu H_{1} H_{2}$. Since the $\mu$ parameter explicitly breaks conformal symmetry, the $B$ parameter is fixed to be $(4 \pi)^{2} M=m_{3 / 2}$ as we found in the previous section. We solve the equations for $\mu$ and $D_{Y}$ as follows:

$$
\begin{aligned}
\mu & =\frac{1}{2 \sin 2 \beta}\left[-B \pm \sqrt{B^{2}-2\left(\bar{m}_{H_{1}}^{2}+\bar{m}_{H_{2}}^{2}\right) \sin ^{2} 2 \beta}\right] \\
D_{Y} & =\cos 2 \beta\left[-m_{Z}^{2}-\left(\bar{m}_{H_{1}}^{2}+\bar{m}_{H_{2}}^{2}+2 \mu^{2}\right)\right]-\left(\bar{m}_{H_{1}}^{2}-\bar{m}_{H_{2}}^{2}\right),
\end{aligned}
$$

where $\bar{m}_{H_{1}}^{2}$ and $\bar{m}_{H_{2}}^{2}$ are the contributions from pure anomaly mediation (without $D$-terms). Since the $B$-term is enhanced by a factor of $(4 \pi)^{2}$ compared to the soft masses, we expand the solution in terms of $1 /(4 \pi)^{2}$. Neglecting all the coupling constants except for the top Yukawa coupling and the strong coupling constants, we obtain two solutions [corresponding to the two signs of Eq. (3.15)] that are approximately:

$$
\begin{array}{llll}
\text { Solution 1: } & \mu \sim-\frac{\bar{m}_{H_{2}}^{2} \sin 2 \beta}{2 B}, & & D_{Y} \sim \bar{m}_{H_{2}}^{2}(1-\cos 2 \beta) \\
\text { Solution 2: } & \mu \sim-\frac{B}{\sin 2 \beta} \quad, & & D_{Y} \sim-\frac{2 B^{2} \cos 2 \beta}{\sin ^{2} 2 \beta}
\end{array}
$$

Notice that the $\mu$ parameter is either $\mathcal{O}\left(M /(4 \pi)^{2}\right)$ or $\mathcal{O}\left((4 \pi)^{2} M\right)$ for Solution 1 or 2 , respectively. 
There are two further conditions for a stable electroweak breaking vacuum. The first condition is that $D_{Y}$ must be negative to stabilize the slepton direction [see Eq. (2.11)], i.e., the sleptons must have positive mass squareds. The second condition arises due to the lack of a quartic potential in the $D$-flat direction and therefore the quadratic terms must have positive coefficients,

$$
\bar{m}_{H_{1}}^{2}+\bar{m}_{H_{2}}^{2}+2 \mu^{2}-2|B \mu|>0
$$

This ensures the potential is bounded from below. For Solution 1 given in Eq. (3.17), this condition reduces to

$$
\bar{m}_{H_{2}}^{2}-\left|\bar{m}_{H_{2}}^{2} \sin 2 \beta\right|>0,
$$

which is satisfied when $\bar{m}_{H_{2}}^{2}>0$. Positive $\bar{m}_{H_{2}}^{2}$ can occur once the top Yukawa coupling becomes asymptotically non-free (requiring $\tan \beta \lesssim 1.5$ ). But, positive $\bar{m}_{H_{2}}^{2}$ is in conflict with the first condition since the sign of $D_{Y}$ is positive for any $\tan \beta$.

Solution 2 always satisfies the stability condition in Eq. (3.19) and the sign of $D_{Y}$ is negative for $\tan \beta<1$. However, this solution has its own potential problem. $D_{Y}$ is generically very large, $\mathcal{O}\left((4 \pi)^{4} M^{2}\right)$, as long as $\tan \beta$ is not very close to 1 , and this causes the squarks to get negative mass squareds. We numerically searched for a solution corresponding to Solution 2 with $\tan \beta$ near 1 including the one-loop corrections to the effective potential and we could not find a solution. Even if there was a solution, the lightest Higgs mass vanishes at tree-level in the limit $\tan \beta \rightarrow 1$ and it would be difficult to satisfy the experimental lower bound from the direct searches of the Higgs boson. Therefore, so long as the $1 /(4 \pi)^{2}$ expansion is a good approximation, we find no acceptable solution.

Intriguingly, we did find a solution with $\tan \beta$ very small, $\mathcal{O}(0.3)$. In this case the top Yukawa coupling $f_{t}=m_{t} /(v \sin \beta)(v=174 \mathrm{GeV})$ is large and thus $f_{t}^{4} /(4 \pi)^{2}$ is no longer a good expansion parameter. For large $f_{t}$, the $\bar{m}_{H_{2}}^{2}$ parameter is given by

$$
\bar{m}_{H_{2}}^{2} \sim 18 f_{t}^{4} M^{2}
$$

and hence we cannot neglect the $2 \bar{m}_{H_{2}}^{2} \sin ^{2} 2 \beta$ factor in Eq. (3.15). Similarly, one can show that $D_{Y}$ does become negative for $\tan \beta \lesssim 0.3$. Adding the one-loop corrections to the effective potential is not qualitatively different from the tree-level analysis. An example solution is

$$
M=300 \mathrm{GeV}, \quad \mu=-4251 \mathrm{GeV}, \quad \tan \beta=0.29, \quad D_{Y}=-2.5 M^{2}, \quad D_{B-L}=2.0 M^{2},(3
$$

that has a proper electroweak symmetry breaking vacuum.

The small value for $\tan \beta=0.29$ causes the top Yukawa coupling to be large, $f_{t}\left(M_{Z}\right)=$ 3.5. Such a large top Yukawa coupling is quasi-perturbative and runs into a Landau pole just above the supersymmetry breaking scale (mass of the top squarks). The perturbative MSSM description breaks down and must be replaced by new description such as a theory with composite top quarks and/or Higgs fields. In the low-energy effective theory, the theory above the cutoff scale is of course unknown. Nevertheless, the UV insensitivity of anomaly mediation allows us to calculate the soft breaking parameters in the IR, and therefore does not lose any predictability at low energies. 
It is interesting to estimate the lightest Higgs boson mass $\left(m_{h}\right)$ for the set of parameters in Eq. (3.22) to clarify whether $m_{h}$ is greater than the present experimental lower bound of $114.4 \mathrm{GeV}$ [31]. We calculated $m_{h}$ with the one-loop correction from the (s)top loop diagrams. Although $m_{h}$ is small at tree-level, the one-loop correction is huge because the top squarks are very heavy, 8.6 TeV and $12 \mathrm{TeV}$, due to the large top Yukawa coupling. The resulting lightest Higgs mass turns out to be $176 \mathrm{GeV}$. However, the uncertainly of the calculation is large because of the heavy stops and large $f_{t}$. Although our one-loop analysis is unable to give a definite prediction, we suspect it is not inconsistent with the experimental lower bound. Nevertheless a more extensive (beyond one-loop) analysis is necessary to be definitive.

\section{Electroweak Symmetry breaking in the NMSSM?}

We have found an electroweak symmetry breaking solution in the MSSM, but the model is not UV complete due to the large top Yukawa coupling blowing up just about the supersymmetry breaking scale. The difficulty in the MSSM is caused by the large value of the $B$ parameter that is induced through the explicit breaking of the conformal symmetry by the $\mu$ parameter. This suggests we should consider a model with no explicit mass parameters, such as the NMSSM [32] with superpotential

$$
W=\lambda S H_{1} H_{2}+\frac{h}{3} S^{3}
$$

Since all the soft breaking parameters are loop suppressed, we naturally expect that no large values of the coupling constants will be needed. The $\mu$ parameter in the MSSM is replaced by $\lambda\langle S\rangle$ that generates the Higgsino mass. Despite these good features, we were unable to find a viable set of parameters because the Higgsino mass turns out to be too small.

The origin of the difficulty in the NMSSM is again due to the high predictability of anomaly mediation. The scalar mass parameter for the singlet $m_{S}^{2}$ is always positive. This is because $S$ interacts only through the couplings $h$ and $\lambda$, where $h$ is always asymptotically non-free and $\lambda$ is asymptotically non-free unless it is much smaller than the gauge couplings. Therefore $S \sim 0$ is almost a stable point of the potential. In order for $S$ to acquire a sizable VEV, large values of either the linear or the cubic terms of $S$ in the scalar potential are needed. Such terms can be obtained from trilinear scalar couplings $A_{\lambda}$ and $A_{h}$, but in anomaly mediation these terms will be large only if $\lambda$ and $h$ are also large. Large superpotential couplings $\lambda$ and $h$ induce a larger $m_{S}^{2}$, that then suppresses the VEV. We therefore find that an arbitrarily large VEV of $S$ cannot be obtained due to this correlation induced by anomaly mediation.

We numerically searched for a parameter set by solving stationary conditions. In Fig. 1 we show solutions found with $M=300 \mathrm{GeV}$ in the $(\tan \beta, \lambda S)$-plane. Upon fixing $M$, $\tan \beta$, and $\lambda S$ there are no remaining free parameters in the Higgs sector. In particular, all the solutions shown in the Figure have a small $\lambda S \lesssim 2.2 \mathrm{GeV}$ leading to a tiny chargino mass that is excluded by experimental bounds. Increasing $M$ is of no help since the soft mass $m_{S}^{2} \propto M^{2}$ increases faster than the trilinear scalar coupling $A_{\lambda} \propto M$.

\footnotetext{
ฯ We ignored the momenta of the external lines in the calculation.
} 


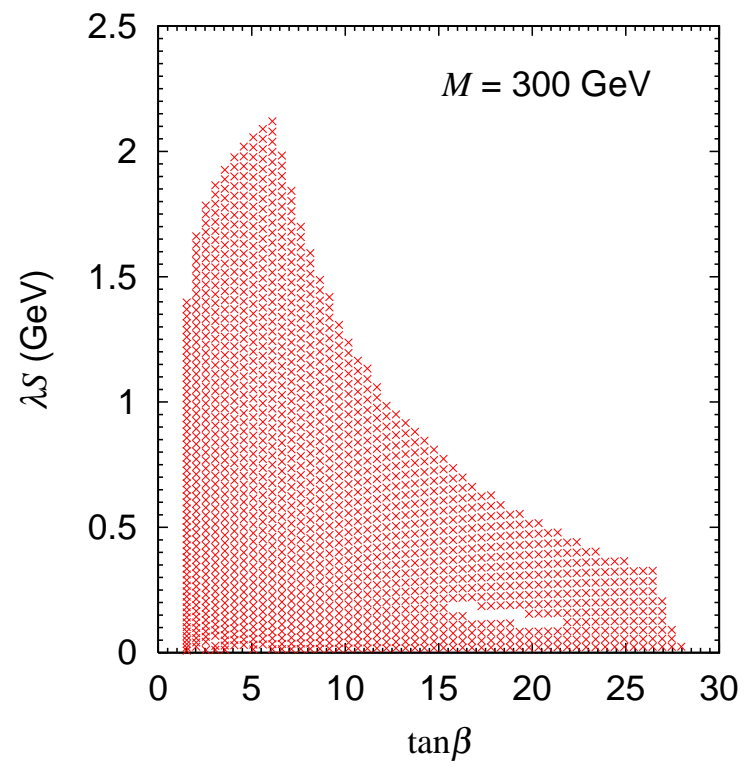

Figure 1: The region of parameters where a stable electroweak symmetry breaking vacuum is found in the NMSSM. The Higgsino mass parameter $\mu=\lambda S$ is too small $\lesssim 3 \mathrm{GeV}$ at all the points.

One possible way to save the NMSSM is to couple the singlet to additional vector-like matter in the superpotential $\lambda^{\prime} S \Phi \Phi$ [33, 34]. The new superpotential coupling $\lambda^{\prime}$ could be asymptotically free if the contribution from the strong coupling dominates. It would be interesting to verify whether this works or not, but the addition of vector-like matter is somewhat ad hoc and so we will not pursue this further in this paper.

\section{$5 \quad$ Electroweak Symmetry Breaking in the Fat Higgs model}

The difficulty of generating a large VEV for $S$ in the NMSSM suggests that a different form of the superpotential is needed. In particular, consider instead

$$
W=\lambda S H_{1} H_{2}+m^{2} S
$$

where $S$ is again a singlet under the SM gauge interactions. This superpotential is the same as the NMSSM superpotential (4.23) with the replacement $k S^{3} / 3 \rightarrow m^{2} S$. The linear term forces $S$ to acquire a large VEV through the soft breaking linear term $C m^{2} S$ (" $C$-term") in the scalar potential.

Interestingly, this superpotential is precisely the low energy effective superpotential of the Minimal Supersymmetric Fat Higgs model [26]. The Fat Higgs model is a very recent idea in which electroweak symmetry is dynamically broken as a result of a new gauge interaction $\mathrm{SU}(2)_{H}$ getting strong at an intermediate scale $\Lambda_{H}$. The minimal field content in the UV theory is three flavors (six doublets) $T^{1 \ldots 6}$ that is known to confine and generate a dynamical superpotential 


$$
W_{\text {dyn. }}=\frac{\operatorname{Pf} M}{\Lambda_{H}^{3}},
$$

for the low energy effective theory of the meson composite fields $M_{i j} \sim T^{i} T^{j}$. The separation between the confinement scale and the electroweak breaking scale is controlled by adding a supersymmetric mass term for one flavor $\left(m_{T} T^{5} T^{6}\right)$. Other superpotential terms are added ensuring that the vacuum aligns to the one with proper electroweak breaking and additional spectator meson composites are lifted. The resulting low energy effective superpotential is then

$$
W_{\text {eff. }}=\lambda M_{56}\left(M_{14} M_{23}-M_{24} M_{13}-v_{0}^{2}\right),
$$

where $v_{0} \simeq \sqrt{m_{T} \Lambda_{H}} / 4 \pi$. This superpotential is identical to Eq. (5.24) upon identifying $M_{56} \rightarrow$ $S,\left(M_{14}, M_{24}\right) \rightarrow H_{1},\left(M_{13}, M_{23}\right) \rightarrow H_{2}$ and $-\lambda v_{0}^{2} \sim m^{2}$. The superpotential (5.24) is therefore UV complete even if the superpotential couplings are large. This will be crucial to be able to find a viable parameter region with electroweak symmetry breaking and no large fine-tuning.

\subsection{The Scalar Potential}

We first discuss the scalar potential and solve the constraints explicitly. In the supersymmetric limit, the superpotential (5.24) forces the VEVs

$$
\left\langle H_{1}^{0}\right\rangle=\left\langle H_{2}^{0}\right\rangle=\sqrt{\frac{-m^{2}}{\lambda}}, \quad\langle S\rangle=0 .
$$

In the presence of the soft supersymmetry breaking effects, the full potential is given by (setting the charged components to zero)

$$
\begin{aligned}
V= & \left|\lambda H_{1}^{0} H_{2}^{0}+m^{2}\right|^{2}+|\lambda|^{2}|S|^{2}\left(\left|H_{1}^{0}\right|^{2}+\left|H_{2}^{0}\right|^{2}\right)+m_{H_{1}}^{2}\left|H_{1}^{0}\right|^{2}+m_{H_{2}}^{2}\left|H_{2}^{0}\right|^{2}+m_{S}^{2}|S|^{2} \\
& +\left[A_{\lambda} S H_{1}^{0} H_{2}^{0}+C m^{2} S+\text { h.c. }\right]+\frac{g_{Y}^{2}+g_{2}^{2}}{8}\left(\left|H_{1}^{0}\right|^{2}-\left|H_{2}^{0}\right|^{2}\right)^{2} .
\end{aligned}
$$

In general $\lambda$ and $m^{2}$ are complex parameters, but we can absorb the phases of these parameters by rotations of $S$ and $H_{1}$ (or $H_{2}$ ). $\|$ Similarly, phase of the soft breaking mass $m_{3 / 2}$ can be absorbed by a $\mathrm{U}(1)_{R}$ rotation. Hence, there are no remaining physical phases that are not proportional to the CKM phase or absorbed into the definition of the strong phase, and therefore this model does not have a supersymmetric CP problem. It is possible that the expectation values of the Higgs fields could have a non-vanishing phase that causes spontaneous CP violation, but we assume we are living in a (sufficiently long-lived [36]) local vacuum that does not break CP. We therefore restrict our discussion to fields with real VEVs of $H_{1}^{0}, H_{2}^{0}$, and $S$.

The soft terms are expressed in terms of the anomalous dimensions by the formulae in Eq. (2.4). In the Fat Higgs model, the anomalous dimension factors are given by

$$
\gamma_{H_{1}}=\frac{1}{(4 \pi)^{2}}\left(\lambda^{2}-\frac{3}{2} g_{2}^{2}-\frac{1}{2} g_{Y}^{2}\right)
$$

\footnotetext{
"This induces a shift in the phase of the determinant of the Yukawa matrices that amounts to shifting the strong CP phase. The strong CP problem is beyond the scope of this work.
} 


$$
\begin{aligned}
\gamma_{H_{2}} & =\frac{1}{(4 \pi)^{2}}\left(\lambda^{2}+3 f_{t}^{2}-\frac{3}{2} g_{2}^{2}-\frac{1}{2} g_{Y}^{2}\right), \\
\gamma_{S} & =\frac{1}{(4 \pi)^{2}} 2 \lambda^{2}, \\
\gamma_{q_{3}} & =\frac{1}{(4 \pi)^{2}}\left(f_{t}^{2}-\frac{8}{3} g_{3}^{2}-\frac{3}{2} g_{2}^{2}-\frac{1}{18} g_{Y}^{2}\right), \\
\gamma_{u_{3}^{c}} & =\frac{1}{(4 \pi)^{2}}\left(2 f_{t}^{2}-\frac{8}{3} g_{3}^{2}-\frac{8}{9} g_{Y}^{2}\right),
\end{aligned}
$$

where $g_{Y}, g_{2}$, and $g_{3}$ are the gauge coupling constants of $\mathrm{U}(1)_{Y}, \mathrm{SU}(2)_{L}$, and $\mathrm{SU}(3)_{C}$, respectively, while $q_{3}$ and $u_{3}^{c}$ represent the left-handed and right-handed up-type quarks of the third generation. The one-loop renormalization group equations for the couplings are given by,

$$
\begin{aligned}
\frac{d}{d t} \lambda & =\lambda\left(\gamma_{H_{1}}+\gamma_{H_{2}}+\gamma_{S}\right), \\
\frac{d}{d t} f_{t} & =f_{t}\left(\gamma_{H_{2}}+\gamma_{q_{3}}+\gamma_{u_{3}^{c}}\right),
\end{aligned}
$$

and by Eq. (2.8) just as in the MSSM. Inserting these beta functions into the expressions for the soft masses, we obtain

$$
\begin{aligned}
m_{H_{1}}^{2}= & \left(4 \lambda^{4}+3 \lambda^{2} f_{t}^{2}-\lambda^{2}\left(3 g_{2}^{2}+g_{Y}^{2}\right)-\frac{3}{2} g_{2}^{4}-\frac{11}{2} g_{Y}^{4}\right) M^{2}+\frac{D_{Y}}{2} \\
m_{H_{2}}^{2}= & \left(4 \lambda^{4}+6 \lambda^{2} f_{t}^{2}+18 f_{t}^{4}-\lambda^{2}\left(3 g_{2}^{2}+g_{Y}^{2}\right)-f_{t}^{2}\left(16 g_{3}^{2}+9 g_{2}^{2}+\frac{13}{3} g_{Y}^{2}\right)\right. \\
& \left.-\frac{3}{2} g_{2}^{4}-\frac{11}{2} g_{Y}^{4}\right) M^{2}-\frac{D_{Y}}{2} \\
m_{S}^{2}= & \left(8 \lambda^{4}+6 \lambda^{2} f_{t}^{2}-\lambda^{2}\left(6 g_{2}^{2}+2 g_{Y}^{2}\right)\right) M^{2} \\
A_{\lambda}= & -\lambda\left(4 \lambda^{2}+3 f_{t}^{2}-3 g_{2}^{2}-g_{Y}^{2}\right) M \\
C= & 2(4 \pi)^{2} M .
\end{aligned}
$$

The $C$-term is $(4 \pi)^{2}$ enhanced because the linear term for $S$ in the superpotential violates the conformal symmetry at tree-level.

There are three minimization conditions to determine the VEVs:

$$
\begin{aligned}
A_{\lambda} S+\lambda m^{2} & =-\frac{\sin 2 \beta}{2}\left(m_{1}^{2}+m_{2}^{2}+\lambda^{2} v^{2}\right) \\
m_{Z}^{2} & =-\frac{m_{1}^{2}-m_{2}^{2}}{\cos 2 \beta}-\left(m_{1}^{2}+m_{2}^{2}\right) \\
0 & =S\left(\lambda^{2} v^{2}+m_{S}^{2}\right)+\frac{1}{2} A_{\lambda} v^{2} \sin 2 \beta+C m^{2}
\end{aligned}
$$

where $m_{1}^{2} \equiv m_{H_{1}}^{2}+\lambda^{2} S^{2}, m_{2}^{2} \equiv m_{H_{2}}^{2}+\lambda^{2} S^{2}$, and $v=\sqrt{\left\langle H_{1}^{0}\right\rangle^{2}+\left\langle H_{2}^{0}\right\rangle^{2}}$. Setting $v=174 \mathrm{GeV}$, we can solve for $S, D_{Y}$, and $m^{2}$ as a function of $\tan \beta$. We obtain

$$
S=\frac{1}{4 \lambda C}\left[\left(m_{S}^{2}+\lambda^{2} v^{2}-\frac{A_{\lambda} C}{\lambda}\right) \frac{2}{\sin 2 \beta}\right.
$$




$$
\left.\pm \sqrt{\left.\left(m_{S}^{2}+\lambda^{2} v^{2}-\frac{A_{\lambda} C}{\lambda}\right)^{2} \frac{4}{\sin ^{2} 2 \beta}+8 A_{\lambda} C \lambda v^{2}-8 C^{2}\left(\bar{m}_{H_{1}}^{2}+\bar{m}_{H_{2}}^{2}+\lambda^{2} v^{2}\right)\right)}\right]
$$

where $\bar{m}_{H_{1}}^{2}$ and $\bar{m}_{H_{2}}^{2}$ are the pure anomaly-mediated soft masses (without $D$-terms). For each solution for $S$, we determine $D_{Y}$ and $m^{2}$ as

$$
\begin{aligned}
D_{Y} & =-\cos 2 \beta\left[m_{Z}^{2}+\bar{m}_{H_{1}}^{2}+\bar{m}_{H_{2}}^{2}+2 \lambda^{2} S^{2}\right]-\left(\bar{m}_{H_{1}}^{2}-\bar{m}_{H_{2}}^{2}\right), \\
m^{2} & =-\frac{\sin 2 \beta}{2 \lambda}\left(\bar{m}_{H_{1}}^{2}+\bar{m}_{H_{2}}^{2}+2 \lambda^{2} S^{2}+\lambda^{2} v^{2}\right)-\frac{A_{\lambda} S}{\lambda} .
\end{aligned}
$$

$D_{Y}$ must be negative to give a positive contributions to both of the slepton soft mass squareds. For $\tan \beta>1$, we find the first term in Eq. (5.45) is positive for $\lambda \sim \mathcal{O}(1)$ while the second term can be sufficiently negative because of the contribution from the strong coupling in Eq. (5.37). For $\tan \beta<1$, the first term is negative while the second term can be positive.

We have four parameters in the Higgs sector $\left(\lambda, m^{2}, M, D_{Y}\right)$. One combination of these parameters is fixed by the $Z$ mass. This leaves three independent parameters that we choose to be $(M, \lambda, \tan \beta)$ with the remainder of the parameters determined by Eqs. (5.44)-(5.46). In Fig. 2, the parameter region where solutions exist in the $(\lambda, \tan \beta)$-plane are shown for a fixed value of $M$. All scalar field mass squareds were required to be positive by choosing the $D_{B-L}$ parameter appropriately. As anticipated above, there are two separate regions with $\tan \beta>1$ and $\tan \beta<1$. Similar regions are obtained for other values of $M$. We should emphasize that it is quite non-trivial to obtain sets of parameters with a stable electroweak breaking vacuum, especially given that there are only three parameters in the model.

The $\tan \beta>1$ case corresponds to the negative-sign solution of Eq. (5.44) while the positivesign solutions are excluded because they imply $D_{Y}>0$. Fig. 目 (left plot) clearly shows that $\lambda$ is fixed to a narrow range around 1 . The lower and upper limits on $\lambda$ can be understood as follows. A small $\lambda$ implies that the pseudo-scalar Higgs mass parameter $m_{1}^{2}+m_{2}^{2}+\lambda^{2} v^{2}$ is small, and this causes a (physical) Higgs mass squared to go negative as we will see in Sec. 5.2. The upper bound on $\lambda$ is due to the requirement $D_{Y}<0$. Also, negative $D_{Y}$ requires $f_{t}$ to be sufficiently asymptotically free. This gives a lower limit of about $\tan \beta \gtrsim 5$.

For $\tan \beta<1$, solutions are found for both signs of Eq. (5.44). Fig. 2 (right plot) shows that $1.4 \lesssim \lambda \lesssim 5$. This region of $\lambda$ is precisely in the range that corresponds to the Fat Higgs model with compositeness at an intermediate scale. The scale of compositeness can be estimated by running $\lambda$ up to its Landau pole. We find it fascinating that a typical value of $\lambda \sim 2$ is perfectly consistent with supersymmetry breaking communicated via UV insensitive anomaly mediation in the Fat Higgs model. As was stressed in [26], there is nothing wrong with a larger $\lambda$ or $f_{t}$ coupling that run into non-perturbative values since the composite Higgs description is expected to break down at an intermediate scale.

The corresponding values of $S$ for these solutions is shown in Fig. 23. For $\tan \beta<1$, the VEV of $S$ is $\mathcal{O}(\mathrm{TeV})$, and thus there is no light Higgsino problem (unlike the NMSSM). The solution with $\tan \beta>1$, however, does generically have a light Higgsino problem. From Eq. (5.41) it is clear that small $S$ is a consistent solution because of the suppression of $\sin 2 \beta$ when $\tan \beta$ is large. The large values of $S$ are obtained only when $M$ is increased, but this also increases the fine-tuning of the Higgs potential for this solution. 

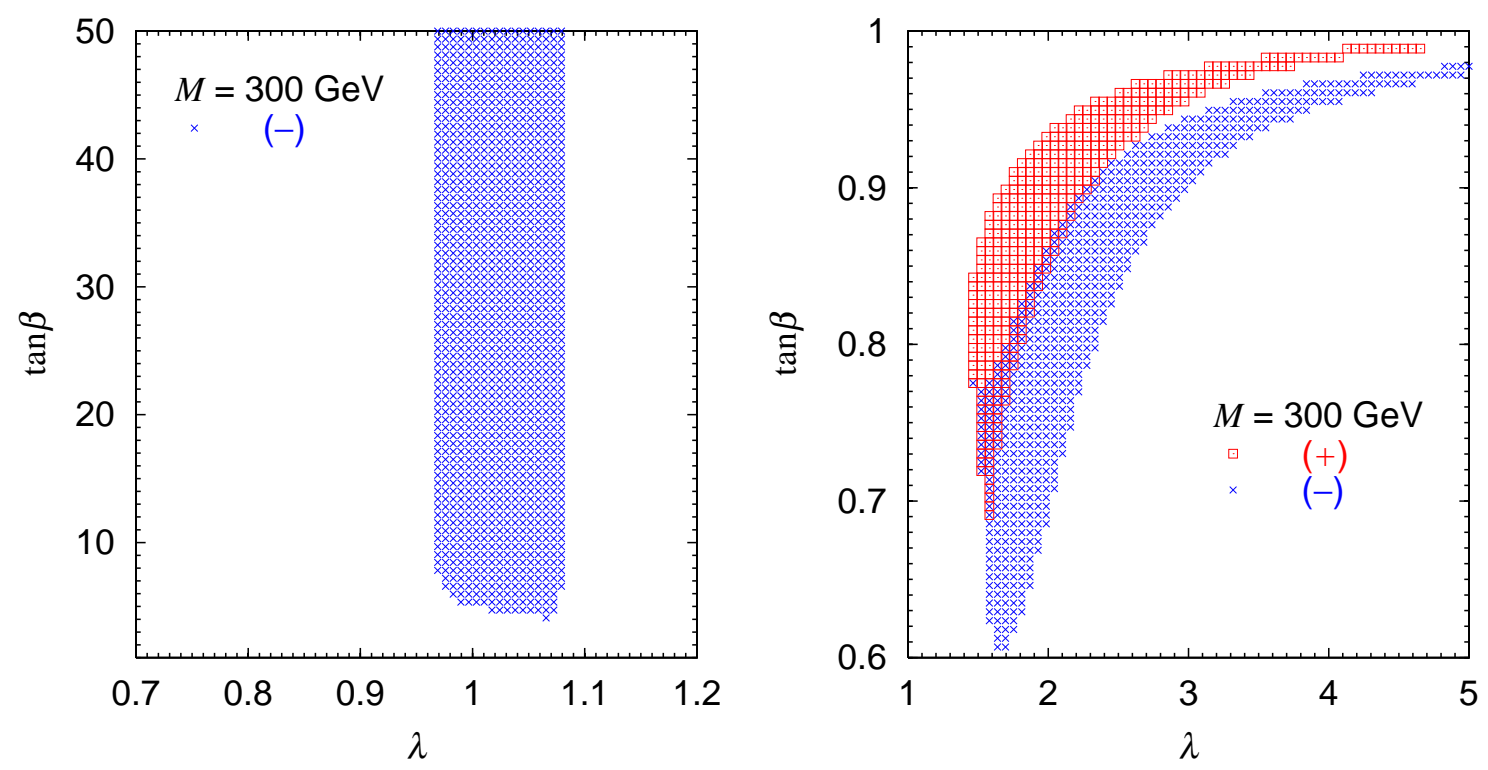

Figure 2: The parameter regions in the Fat Higgs model where electroweak symmetry breaking solutions with all scalar particle mass squareds positive is shown for fixed $M=300 \mathrm{GeV}$. The two plots correspond to the two distinct regions of parameters $\tan \beta>1$ and $\tan \beta<1$. For $\tan \beta<1$, there are two solutions shown by $\square$ and $\times$ that correspond to the positive and negative sign solution to Eq. (5.44), respectively.

\subsection{The Mass Spectrum}

The Fat Higgs model with UV insensitive anomaly-mediated soft breaking has a mass spectrum that is distinctly different from the MSSM. Here we present the mass matrices of the superpartners and discuss the general features of the spectrum.

We first consider the Higgs boson mass. There are three CP-even Higgs bosons in the model. The mass matrix in the basis $\left(H_{1}^{0}, H_{2}^{0}, S\right)$ is given by

$$
\left(\begin{array}{ccc}
m_{Z}^{2} \cos ^{2} \beta+m_{X}^{2} \sin ^{2} \beta & * & * \\
-\left(m_{X}^{2}+m_{Z}^{2}-2 \lambda^{2} v^{2}\right) \sin \beta \cos \beta & m_{Z}^{2} \sin ^{2} \beta+m_{X}^{2} \cos ^{2} \beta & * \\
\left(A_{\lambda} \sin \beta+2 \lambda^{2} S \cos \beta\right) v & \left(A_{\lambda} \cos \beta+2 \lambda^{2} S \sin \beta\right) v & m_{S}^{2}+\lambda^{2} v^{2}
\end{array}\right)
$$

where $m_{X}^{2}=m_{1}^{2}+m_{2}^{2}+\lambda^{2} v^{2}$ and the off-diagonal entries $*$ are suppressed using the obvious symmetry of the matrix. In the limit where $M \gg v$ and/or $\lambda \gg 1$, we can ignore the mixing between the singlet and the doublet Higgses. If we consider the $2 \times 2$ sub-matrix of the doublet Higgses, the eigenvalues are given by

$$
\begin{aligned}
& m_{H^{0}, h^{0}}^{2} \sim \frac{1}{2}\left[m_{Z}^{2}+m_{X}^{2}\right. \\
&\left.\quad \pm \sqrt{\left(m_{Z}^{2}-m_{X}^{2}\right)^{2}+4 m_{Z}^{2} m_{X}^{2} \sin ^{2} 2 \beta-4 \lambda^{2} v^{2}\left(m_{Z}^{2}+m_{X}^{2}+\lambda^{2} v^{2}\right) \sin ^{2} 2 \beta}\right]
\end{aligned}
$$


(a)

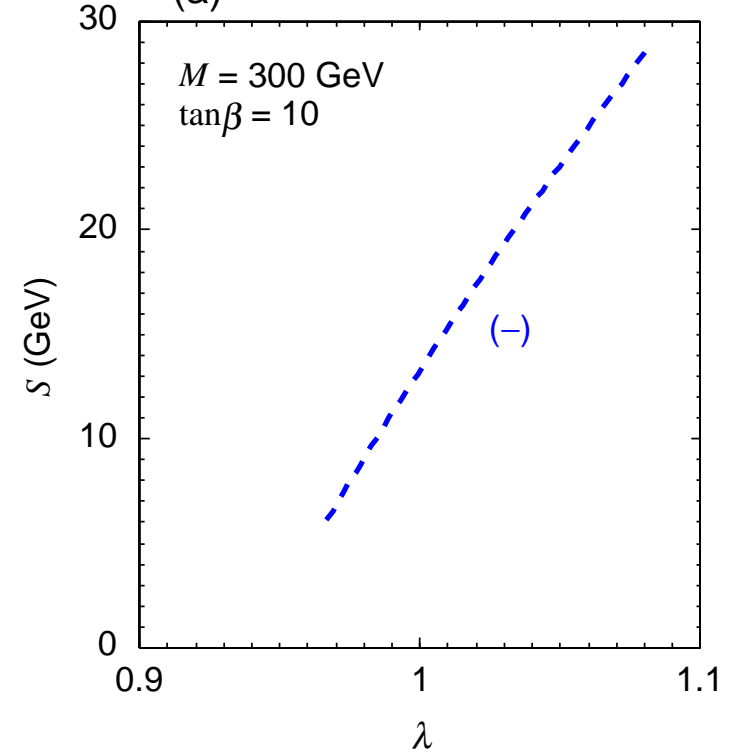

(b)

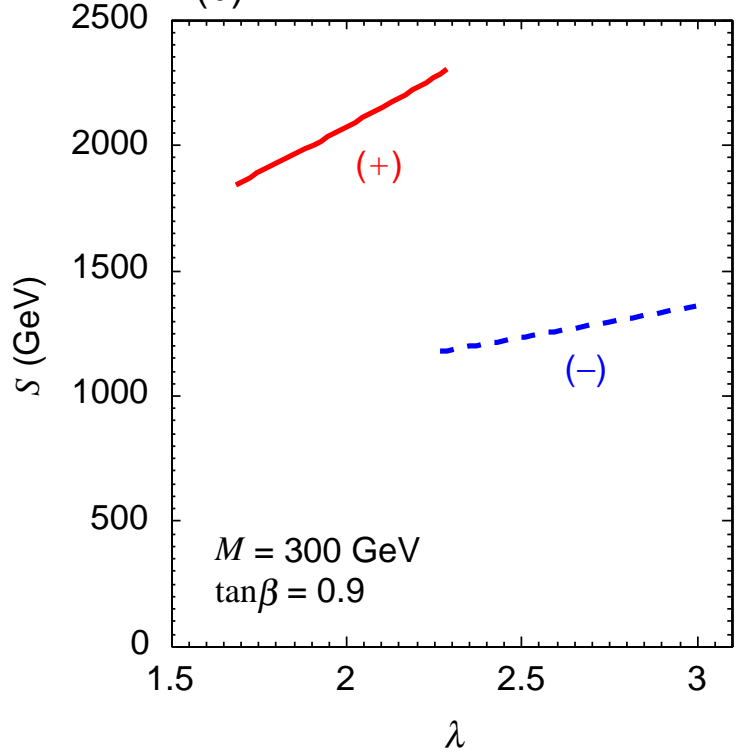

Figure 3: The expectation values of $S$ are shown as functions of $\lambda$ with fixed $M=300 \mathrm{GeV}$ and $\tan \beta=10$ [plot (a)] and 0.9 [plot (b)].

From the above formula, we see that the lightest Higgs mass approaches $m_{Z}$ when $\tan \beta \gg 1$ or $\tan \beta \ll 1$. Whether such a parameter choice is phenomenologically viable depends on adding the usual radiative corrections to the Higgs mass [37, 38, 39]. For $\tan \beta \sim 1, m_{h^{0}}$ can be much larger than $m_{Z}$. This is an interesting feature of the Fat Higgs model.

In the same basis, the mass matrix for the CP-odd Higgs bosons is given by

$$
\left(\begin{array}{ccc}
m_{X}^{2} \sin ^{2} \beta & m_{X}^{2} \sin \beta \cos \beta & -A_{\lambda} v \sin \beta \\
m_{X}^{2} \sin \beta \cos \beta & m_{X}^{2} \cos ^{2} \beta & -A_{\lambda} v \cos \beta \\
-A_{\lambda} v \sin \beta & -A_{\lambda} v \cos \beta & m_{S}^{2}+\lambda^{2} v^{2}
\end{array}\right) .
$$

After rotating the (12)-plane by the angle $\beta$, the matrix becomes

$$
\left(\begin{array}{ccc}
0 & 0 & 0 \\
0 & m_{X}^{2} & -A_{\lambda} v \\
0 & -A_{\lambda} v & m_{S}^{2}+\lambda^{2} v^{2}
\end{array}\right)
$$

The massless mode corresponds to the would-be Nambu-Goldstone boson eaten by the $Z$ boson. We can clearly see that the mass parameter $m_{X}^{2}$ has to be at least positive in order for the potential to be stable. That gives the lower limit of $\lambda$ for solutions with $\tan \beta>1$.

The charged Higgs boson mass is given by

$$
m_{H^{ \pm}}^{2}=m_{1}^{2}+m_{2}^{2}+m_{W}^{2},
$$

that is reminiscent of the MSSM $\left.m_{H^{ \pm}}^{2}\right|_{\mathrm{MSSM}}=2 \mu^{2}+m_{H_{1}}^{2}+m_{H_{2}}^{2}+m_{W}^{2}$. 
Charginos have a similar mass matrix to that in the MSSM:

$$
\left(\begin{array}{cc}
M_{2} & -g_{2} v \cos \beta \\
-g_{2} v \sin \beta & \lambda S
\end{array}\right),
$$

where $M_{2}$ is the gaugino mass of $\mathrm{SU}(2)_{L}$ given by $M_{2}=-g_{2}^{2} M$. The charginos are heavier than the experimental bounds from LEP so long as the Higgsino mass parameter is $\lambda S \gtrsim \mathcal{O}(100 \mathrm{GeV})$, and the supersymmetry breaking parameter $M \gtrsim 250 \mathrm{GeV}$.

The neutralino sector is very different from the MSSM. There is an additional neutralino $\left(\psi_{S}\right.$ : singlino) from the fermionic component of $S$. The mass matrix is given in the basis $\left(\tilde{B}^{0}\right.$, $\left.\tilde{W}^{0}, \tilde{H}_{1}^{0}, \tilde{H}_{2}^{0}, \psi_{S}\right)$ as follows:

$$
\left(\begin{array}{ccccc}
M_{1} & * & * & * & * \\
0 & M_{2} & * & * & * \\
g_{Y} v \cos \beta / \sqrt{2} & -g_{2} v \cos \beta / \sqrt{2} & 0 & * & * \\
-g_{Y} v \sin \beta / \sqrt{2} & g_{2} v \sin \beta / \sqrt{2} & -\lambda S & 0 & * \\
0 & 0 & -\lambda v \sin \beta & -\lambda v \cos \beta & 0
\end{array}\right)
$$

where $M_{1}=-11 g_{Y}^{2} M$ is the gaugino mass of $\mathrm{U}(1)_{Y}$. The singlino mass is induced only through the mixing with the Higgsinos.

The formulae for the sfermion masses are the same as the MSSM by replacing the $\mu$ parameter with $\lambda S$. Positive slepton mass squareds require (2.11) to be satisfied, and there is an upper bound on $\left|D_{Y}\right|$ to ensure the squark mass squareds do not become negative. An interesting feature is that the stops become much heavier than the other sfermions when $\tan \beta<1$, since the asymptotically non-free top Yukawa coupling gives large positive contributions to the stop masses. This is a distinctly different feature from other supersymmetry breaking scenarios.

\subsection{Examples of Viable Parameters}

Here we demonstrate explicitly the viability of UV insensitive anomaly mediation with the Fat Higgs model superpotential, by presenting sample sets of parameters with our computations of the soft breaking parameters. From Sec. 5.1, we know stable electroweak breaking vacua exist for two separate regions of the parameter space: $\tan \beta<1$ and $\tan \beta>1$.

In Table 1 we present sample parameters and the computed spectra for $\tan \beta<1$ (Point I) and $\tan \beta>1$ (Point II). The input parameters include the supersymmetric parameters $\lambda$ and $m^{2}$, and the supersymmetry breaking parameters $M, D_{Y}$ and $D_{B-L}$. Everything is calculated from these five parameters. $\tan \beta$ and $S$ are fixed at the minimum of the potential, while the remainder of Table 11 provides the masses of the superpartners.

Point I is perhaps the most interesting case. At tree-level, the lightest Higgs boson mass is much larger than $m_{Z}$ because of the quartic term in the potential has a coefficient of $\lambda^{2}$ [26]. The lightest supersymmetric particle (LSP) is the lightest neutralino that is mostly a singlino, and thus the lower bound from the direct search experiments does not apply. Other than the lightest Higgs boson, the particles in the Higgs sector are several $\mathrm{TeV}$ due to the large value of $\lambda$. 


\begin{tabular}{|c|c|c|c|}
\hline & & $\begin{array}{l}\text { Point I } \\
(\tan \beta<1)\end{array}$ & $\begin{array}{l}\text { Point II } \\
(\tan \beta>1)\end{array}$ \\
\hline \multirow{5}{*}{$\begin{array}{l}\text { input } \\
\text { parameters }\end{array}$} & $\lambda$ & 2.3 & 1.05 \\
\hline & $m^{2}\left[\mathrm{GeV}^{2}\right]$ & $-3.1 \times 10^{5}$ & $-4.8 \times 10^{3}$ \\
\hline & $M[\mathrm{GeV}]$ & & 900 \\
\hline & $D_{Y}\left[\mathrm{GeV}^{2}\right]$ & $-2.8 M^{2}$ & $-2.9 M^{2}$ \\
\hline & $D_{B-L}\left[\mathrm{GeV}^{2}\right]$ & $2 M^{2}$ & $2 M^{2}$ \\
\hline \multirow{27}{*}{$\begin{array}{l}\text { output } \\
\text { results }\end{array}$} & $\tan \beta$ & 0.9 & 5.0 \\
\hline & $S[\mathrm{GeV}]$ & 1186 & 133 \\
\hline & $m_{H_{1}^{0}}[\mathrm{GeV}]$ & 355 & 47 \\
\hline & $m_{H_{2}^{0}}[\mathrm{GeV}]$ & 4976 & 1930 \\
\hline & $m_{H_{3}^{0}}^{2}[\mathrm{GeV}]$ & 6614 & 3258 \\
\hline & $m_{A_{1}^{0}}[\mathrm{GeV}]$ & 4924 & 1926 \\
\hline & $m_{A_{2}^{0}}^{1}[\mathrm{GeV}]$ & 6662 & 3260 \\
\hline & $m_{H^{ \pm}}[\mathrm{GeV}]$ & 6613 & 1954 \\
\hline & $m_{\chi_{1}^{ \pm}}[\mathrm{GeV}]$ & 128 & 140 \\
\hline & $m_{\chi_{2}^{ \pm}}[\mathrm{GeV}]$ & 2730 & 394 \\
\hline & $m_{\chi_{1}^{0}}[\mathrm{GeV}]$ & 57 & 44.6 \\
\hline & $m_{\chi_{2}^{0}}[\mathrm{GeV}]$ & 128 & 214 \\
\hline & $m_{\chi_{3}^{0}}[\mathrm{GeV}]$ & 419 & 241 \\
\hline & $m_{\chi_{4}^{0}}[\mathrm{GeV}]$ & 2731 & 394 \\
\hline & $m_{\chi_{5}^{0}}[\mathrm{GeV}]$ & 2785 & 1257 \\
\hline & $m_{\tilde{l}_{L}}[\mathrm{GeV}]$ & 147 & 423 \\
\hline & $m_{\tilde{e}_{R}}[\mathrm{GeV}]$ & 202 & 640 \\
\hline & $m_{\tilde{\tau}_{1}}[\mathrm{GeV}]$ & 144 & 422 \\
\hline & $m_{\tilde{\tau}_{2}}[\mathrm{GeV}]$ & 205 & 638 \\
\hline & $m_{\tilde{u}_{L}}[\mathrm{GeV}]$ & 1241 & 3724 \\
\hline & $m_{\tilde{u}_{R}}[\mathrm{GeV}]$ & 1209 & 3622 \\
\hline & $m_{\tilde{t}_{1}}[\mathrm{GeV}]$ & 1689 & 3026 \\
\hline & $m_{\tilde{t}_{2}}[\mathrm{GeV}]$ & 2131 & 3444 \\
\hline & $m_{\tilde{d}_{L}}[\mathrm{GeV}]$ & 1241 & 3725 \\
\hline & $m_{\tilde{d}_{R}}[\mathrm{GeV}]$ & 1313 & 3941 \\
\hline & $m_{\tilde{b}_{1}}[\mathrm{GeV}]$ & 1313 & 3441 \\
\hline & $m_{\tilde{b}_{2}}[\mathrm{GeV}]$ & 1728 & 3929 \\
\hline
\end{tabular}

Table 1: Two sample sets of parameters and the resulting superpartner masses are shown. Note that these are tree-level values; radiative corrections are important especially for the lightest Higgs mass. 
The large $\lambda$ and $f_{t}$ indicate the existence of strong coupling well above the electroweak scale. This is precisely what is expected in the Fat Higgs model when the Higgs and singlet superfields' compositeness is revealed. Indeed, large $\lambda$ and the existence of a linear term in $S$ is a prediction of the low energy effective theory of the Fat Higgs model.

The second column shown as Point II in Table 1 provides a sample spectrum with $\tan \beta>1$. As was anticipated earlier, the solution with $\tan \beta>1$ requires large $M$ to have sufficiently heavy Higgsinos. Note that the tree-level mass for the lightest Higgs boson of $47 \mathrm{GeV}$ is rather light but is expected to be modified by a large radiative correction from the (s)top-loop and $S$-loop diagrams. Parameterizing the loop contributions as

$$
m_{H_{1}^{0}}^{2}=\left(m_{H_{1}^{0}}^{\text {tree }}\right)^{2}+\Delta_{t}+\Delta_{S}
$$

we obtain

$$
\Delta_{t} \sim \frac{3}{4 \pi^{2}}\left(\frac{m_{t}^{4}}{v^{2}}\right) \log \frac{m_{\tilde{t}}^{2}}{m_{t}^{2}} \sim(105 \mathrm{GeV})^{2}
$$

that should be large enough to push the Higgs mass above the LEP bound.

\subsection{Phenomenology}

The main general predictions of the anomaly mediation are: (1) A relatively large difference in mass between the colored superpartners (gluino, squarks) and the uncolored ones (sleptons, neutralinos, charginos), (2) the characteristic prediction on the gaugino masses in proportion to their beta functions, and (3) the heavy gravitino. Next, we know the superpotential given in Eq. (5.24) is UV completed by the Fat Higgs model [26] that also determines electroweak symmetry breaking. In this framework we found two distinct regions of parameters represented by Points I and II given in Table 1 that we will use for the discussion of the phenomenology.

For the region of parameters characterized by $\tan \beta \lesssim 1$, represented by Point I, we found (1) a large effective $\mu$-parameter, and (2) a light neutralino that is mostly a singlino. For the region of parameters characterized by $\tan \beta \sim 5$, represented by Point II, we found (1) the effective $\mu$ parameter at the weak scale, and (2) a light neutralino that is mostly a Higgsino. These general features lead to interesting and quite distinct phenomenology and cosmology from conventional supersymmetric models with minimal supergravity or its slight modifications.

The first observation is that the Fat Higgs model with UV insensitive anomaly mediation contains heavy squark and gluino masses yet only a mild fine-tuning comparable to that in the MSSM (discussed in the next section). Unlike the MSSM, however, the flavor problem as well as the supersymmetric CP problem are completely solved due to the UV insensitivity.

The LHC is expected to discover supersymmetry for Point I through missing energy and dilepton signatures of squarks and gluinos, although their heaviness limits the event rate and hence precision measurements will not be easy. Nonetheless $M_{3}$ should be measured fairly well [40]. The chargino and the second neutralino are basically winos, and the sleptons are light. The trilepton signature is a target at Tevatron. At LHC, both the trilepton signature of chargino/neutrlino and dilepton signature of sleptons can be searched for. For Point II, squarks 
and gluinos may be beyond the reach of the LHC. On the other hand, both chargino states and four neutralino states are relatively light and can be looked for. In anomaly-mediated models studied in the literature with the addition of a UV sensitive universal scalar mass, the lighter chargino and the lightest neutralino are mostly wino and have very small mass splitting . In our UV insensitive model, by contrast, the lighter chargino decays into the lightest neutralino with a sizable mass splitting and thus the chargino signal becomes a good one to seek.

The uncolored states likely can be produced at an $e^{+} e^{-}$Linear Collider of $\sqrt{s}=0.5-1 \mathrm{TeV}$. The most important measurement is the chargino and neutralino compositions and the extraction of $M_{1}$ and $M_{2}$ 41. Combining the LHC and LC data, it seems feasible to experimentally verify the main quantitative prediction of anomaly mediation that is the ratios of gaugino masses (it may leave an ambiguity with a particular type of gauge-mediated supersymmetry breaking [42], but this can be resolved by studying the scalar masses).

The Higgs spectrum is quite distinct from conventional supersymmetry. There is a Standard Model-like Higgs that is relatively heavy in Point I. In this mass range, the Higgs mostly decays into $W W$ and $Z Z$ that makes its discovery and further study quite easy at the LHC. It has another potential invisible decay mode into a pair of the lightest neutralinos. Fortunately, the lightest neutralino has little Higgsino component and hence the invisible branching fraction is suppressed. Therefore, the LHC can find not only the Higgs but also determine its width, its spin, and couplings to $W W$ and $Z Z$ at $10 \%$ level. For Point II, the tree-level Higgs mass is light but the radiative correction pushes it above the current LEP bound. In this case the invisible decay mode into a pair of the lightest neutralino becomes important, and the search may be difficult. Nonetheless the $W$-fusion process with double forward jet tagging will be helpful [43]. On the other hand, the Higgs would definitely be found at an $e^{+} e^{-}$Linear Collider. For both Points, verifying the model experimentally is difficult because the other Higgs states are all very heavy, in the multi-TeV range, and they are only pair-produced. The only potential sensitivity is in the precision electroweak measurement, i.e. at GigaZ, where an additional contribution to the $T$-parameter from the heavy Higgs states may be extracted. Much higher energy colliders such as VLHC and/or CLIC will be needed to directly probe these states.

The scalar mass spectrum is determined by only three parameters, the overall scale and two $D$-terms, and thus has many non-trivial sum rules. Gaugino masses determine $M$, while left- and right-handed sleptons determine two $D$-terms, and together with $m_{H}$, there are no remaining free parameters. The remainder of the superpartner spectrum could then be predicted and searched for at colliders. Therefore precise measurements of squark masses would allow nontrivial tests of the model. This is a task for a very high-energy $e^{+} e^{-}$linear collider such as CLIC.

One potential concern is the invisible width of $Z$-boson for the Point II, as $Z$-boson may decay into a neutralino pair. For this particular set of parameters the invisible width is modified by less than $1 \%$ of an additional neutrino species, and is completely acceptable. Nevertheless, in general there is a significant constraint on the region of parameters with $\tan \beta \gtrsim 5$ because we find the mass of the lightest neutralino tends to be less than but of order $m_{Z} / 2$.

Finally, a few comments on neutrinos and lepton flavor violation are in order. UV insensitive anomaly mediation naturally incorporates right-handed neutrinos. This is simply because three generations of right-handed neutrinos are required to gauge $\mathrm{U}(1)_{B-L}$ that itself is needed to 
allow the $D$-term for $\mathrm{U}(1)_{B-L}$. There are several possibilities for generating neutrino masses [22, 23]:

- Forbid the usual $\lambda_{\nu} L H_{2} \nu^{c}$ term in the superpotential but include the higher dimension operator $\lambda_{\nu} L H_{2} \nu^{c} / M_{\mathrm{Pl}}$ in the Kähler potential [22]. Inserting appropriate powers of the compensator and expanding this out one obtains a Yukawa coupling of order $m_{3 / 2} / M_{\mathrm{Pl}}$, allowing for Dirac neutrinos of about the right scale while preserving UV insensitivity.

- The usual see-saw mechanism 44] with order one Yukawa couplings $\lambda_{\nu} L H_{2} \nu^{c}$ and a large right-handed neutrino mass scale $\left[\mathrm{U}(1)_{B-L}\right.$ breaking scale] generated dynamically [23]. This will induce UV sensitive corrections to the lepton sector of the low energy theory, but the leading order result remains unaffected.

If the usual see-saw mechanism is adopted, the deflection from the UV insensitive trajectory would lead to potentially observable lepton flavor violating processes, such as $\mu \rightarrow e \gamma$. This is not required, of course, since the right-handed neutrino mass scale could be much lower with smaller neutrino Yukawa couplings.

\subsection{Cosmology}

Several cosmological implications of anomaly mediation with UV insensitivity are quite interesting. The gravitino problem of supersymmetry are practically solved because it decays before the Big Bang Nucleosynthesis [45, 46]. The gravitino is expected to decay into the lightest supersymmetric particle and other particles with hadronic or electromagnetic energy that thermalize quickly. Therefore, the only constraint is that the gravitino is not too abundant before their decay so as not to overclose the universe through the LSP decay product. This consideration $\left(\Omega_{\chi} \leq 1\right)$, applied to gravitinos, leads to an upper bound on the reheating temperature 47]

$$
T_{R} \leq 2.7 \times 10^{11} \mathrm{GeV}\left(\frac{100 \mathrm{GeV}}{m_{\chi}}\right) h^{2} .
$$

Rescaling to the dark matter density measured by WMAP, $\Omega h^{2} \simeq 0.11$ [48] the revised constraint is

$$
T_{R} \leq 3.0 \times 10^{10} \mathrm{GeV}\left(\frac{100 \mathrm{GeV}}{m_{\chi}}\right) .
$$

Note that if the reheating temperature is close to the upper bound, the neutralino can give the correct abundance even if the annihilation cross section is too large.

Interestingly, the large allowed reheating temperature (due to the large gravitino mass) allows thermal leptogenesis. For a sub-TeV gravitino, the reheating temperature is required to be less than $10^{6}-10^{9} \mathrm{GeV}$ [47], while thermal leptogenesis prefers $T_{R H} \gtrsim 10^{10} \mathrm{GeV}$ 49, 50]. Even though strict UV insensitivity requires right-handed sneutrinos to have a mass at the electroweak scale, they can be made heavy as in the conventional seesaw mechanism with potentially observable lepton-flavor violation as discussed in the previous subsection. In this case, the thermal leptogenesis would be viable. 
As for dark matter, UV insensitive anomaly mediation predicts the LSP is the lightest neutralino that is mostly a singlino or a Higgsino. In general this allows the mass of the LSP to be smaller in comparison with conventional models. The lightest neutralino acquires its mass due to a see-saw like structure in the neutralino mass matrix. To a good approximation, we can ignore the mixing with the gaugino states for both regions of parameters. For Point I, the two neutral Higgsino states have a vector-like mass of $\mu=\lambda S$, while the determinant in the lower three-by-three matrix is $2 \lambda^{2} \mu v^{2} \sin \beta \cos \beta$. Therefore the lightest neutralino has a mass of approximately $m_{\chi_{1}^{0}} \simeq 2 \lambda^{2} v^{2} \sin \beta \cos \beta / \mu$ and its wave function is

$$
\chi_{1}^{0} \simeq \tilde{S}-\frac{\lambda v \cos \beta}{\mu} \tilde{H}_{u}^{0}-\frac{\lambda v \sin \beta}{\mu} \tilde{H}_{d}^{0}
$$

Despite the large $\mu$, however, the mixing is not so small given a large $\lambda v$. When $\tan \beta \approx 1$, the $H_{u}^{0}$ and $H_{d}^{0}$ components in $\tilde{\chi}_{1}^{0}$ cancel in the coupling to the $Z$-boson, while the annihilation due to the Higgs exchange is also small due to the large Higgs mass and its $P$-wave nature. The annihilation cross section is of the order of $10^{-12} \mathrm{GeV}^{-2}$, while the correct abundance is obtained for $\left\langle\sigma_{a n n} v_{r e l}\right\rangle \simeq 10^{-9} \mathrm{GeV}^{2}$. The neutralino is therefore overabundant in Point I. However, there are viable parameter solutions in which the mass of the lightest neutralino is much closer to $m_{Z} / 2$, and therefore the annihilation cross section is significantly enhanced by the $Z$-pole in the $P$-wave. A completely separate possibility is that a would-be overabundant singlino is diluted by entropy production below the electroweak scale. Conversely, for Point II the lightest neutralino has a large Higgsino component (66.5\%) and a singlino component (33.3\%). Then the $Z$-exchange in the $s$-channel is large due to the large number of final states despite its $P$-wave nature. The annihilation cross section is of the order of $10^{-7} \mathrm{GeV}^{-2}$. Obviously the precise relic abundance of any particular set of parameters must be calculated carefully, but we are happy to find that the abundance varies around the cosmologically interesting value and the lightest neutralino ought to be a good dark matter candidate.

The moduli fields expected in most hidden sector models of supersymmetry breaking as well as string theory acquire their masses from supersymmetry breaking of the order of $m_{3 / 2}$, and decay with a lifetime comparable to that of the gravitino. They are typically displaced from the minimum with an $\mathcal{O}\left(M_{P l}\right)$ amplitude, dominating the universe before they decay, making Big Bang Nucleosynthesis (BBN) impossible. They also dilute any preexisting baryon asymmetry by $\mathcal{O}\left(M_{P l} / m_{3 / 2}\right)$. This has been the major embarrassment of the hidden sector models [51] or string theory [52]. In our case, on the other hand, they decay before BBN, a major improvement in supersymmetric cosmology.

There are remaining concerns, however. For instance, if the moduli decay copiously into superparticles, they will eventually cascade down to the lightest supersymmetric particle (LSP) that would overclose the Universe (assuming the LSP is stable and has an electroweak-scale mass) [53, 54].** One possibility is that the LSP has a mass below about $1 \mathrm{GeV}$ and does not overclose the universe. It was argued [55] that the axino, the fermionic superpartner of the invisible axion, is a possible candidate of a light LSP in the context of the gauge-mediated supersymmetry breaking. In a typical gravity-mediated supersymmetry breaking, the axino acquires a mass of $\mathcal{O}\left(m_{3 / 2}\right)$ and this idea does not work [56]. In anomaly mediation, however,

${ }^{* *}$ The branching fractions of the moduli are certainly highly model-dependent and may not copiously produce superparticles. 
the axion lacks renormalizable couplings in the infrared and hence its mass is indeed suppressed by the inverse power of the decay constant. The axino is therefore a viable candidate for the LSP. Yet another possibility is that the $R$-parity is broken and the LSP decays.

Of course the moduli may simply not exist, or equivalently, may had been fixed in a supersymmetric fashion. Recent string theory compactifications with anti-symmetric tensor field background together with D-branes are shown to be capable of indeed doing so [57, 58]. Hidden sector models can avoid light moduli if the supersymmetry breaking is dynamical [59], or of O'Raifeartaigh type instead of Polonyi-like [60, 61]. There is a possibility that the baryon asymmetry may be generated by the gravitino decay [62], which may be generalized to the moduli decay.

\subsection{Fine Tuning?}

The mass parameters in the Higgs sector appear alarmingly large because of the large values of $\lambda$ and $f_{t}$, suggesting some fine-tuning among the parameters is needed to obtain the small value of $v$. Here we estimate the amount of fine-tuning for each case and find that it is no worse than the MSSM with minimal supergravity.

The Higgs VEV $v$ is approximately solved by expanding Eqs. (5.41) and (5.42) in terms of $v^{2} / M^{2}$ as follows:

$$
\begin{aligned}
v^{2} & \sim \frac{m_{1}^{2}+m_{2}^{2}}{2 \lambda^{2}}\left[1-\frac{m_{1}^{2} m_{2}^{2}}{\left(A_{\lambda} S+\lambda m^{2}\right)^{2}}\right] \\
& \sim \frac{m_{1}^{2}+m_{2}^{2}}{2 \lambda^{2}}\left[1-\left(\frac{\lambda}{\lambda^{2}+(4 \pi)^{2}}\right)^{2} \frac{m_{1}^{2} m_{2}^{2}}{m^{4}}\right],
\end{aligned}
$$

where in the second expression we used an approximate solution of $S \sim-C m^{2} / m_{S}^{2}$ determined by Eq. (5.43). Ignoring all the couplings except for $\lambda$, the prefactor of $\left(m_{1}^{2}+m_{2}^{2}\right) /\left(2 \lambda^{2}\right)$ is estimated to be $4 \lambda^{2} M^{2}+S^{2}$ that is much larger than $v^{2}$ for the solutions discussed in Sec. 5.1. Therefore a cancellation between two terms in the parentheses is necessary. It is important to remark that this fine-tuning resulted because anomaly mediation is communicating supersymmetry breaking to the soft masses. Fine-tuning would be eliminated if soft supersymmetry breaking were fixed independent of raising of $\lambda$, as originally found in [26], but this is not possible in anomaly mediation because $m_{1}^{2}+m_{2}^{2}$ contains a term proportional to $\lambda^{4}$.

Fortunately the typical value of fine-tuning is $\mathcal{O}(1 \%)$, no worse than the MSSM 63. This is quite surprising given that the spectrum is much heavier than the MSSM with gravity mediation. The reason why the fine-tuning is smaller is because the Higgs VEV is mainly determined by the potential with coupling $\lambda$ and not the gauge couplings. The corresponding formula in the MSSM is given by

$$
v^{2} \sim \frac{4\left(m_{1}^{2}+m_{2}^{2}\right) B^{2} \mu^{2}}{\left(g_{Y}^{2}+g_{2}^{2}\right)\left(m_{1}^{2}-m_{2}^{2}\right)^{2}}\left[1-\frac{m_{1}^{2} m_{2}^{2}}{B^{2} \mu^{2}}\right] .
$$

Clearly a more accurate cancellation is necessary for the MSSM as compared to Eq. (5.59) because of the small gauge coupling constants, even when all the supersymmetry breaking parameters are of the same order. 


\section{Conclusions}

We found a stable electroweak symmetry breaking vacuum with supersymmetry breaking communicated via UV insensitive anomaly mediation. In the MSSM we found that this occurs when $\tan \beta \sim 0.3$; no solutions for $\tan \beta>1$ were found. This implies the top Yukawa coupling blows up slightly above the supersymmetry breaking scale. The MSSM with UV insensitive anomaly mediation is therefore UV incomplete, even though (by definition) this does not affect the predictions on the supersymmetry breaking parameters. We searched the parameter space of the NMSSM and were unable to find a viable set of parameters due to the prediction of tiny chargino masses that are ruled out by direct search experiments.

We did find a stable vacuum and viable spectrum for a model with a modified (NMSSM-like) superpotential in which the the cubic singlet term is replaced with a term linear in the singlet. Two distinct regions of parameter space were found: $\tan \beta \lesssim 1$ and $\tan \beta \gtrsim 5$. The parameter region with $\tan \beta \lesssim 1$ requires the $S H_{1} H_{2}$ coupling to be $\lambda \gtrsim 1.5$, that is remarkably just what is expected in the "Fat Higgs" model that generates this NMSSM-like superpotential dynamically. The first UV complete, UV insensitive supersymmetry breaking model with a stable electroweak symmetry breaking vacuum is therefore the Fat Higgs model with UV insensitive anomaly mediation.

We also found a viable parameter region with $\tan \beta \gtrsim 5$. In this case, $\lambda \simeq 1.0$ and the Higgs is "less fat." Since $\tan \beta$ is moderately large, the Higgs potential is similar to that in the MSSM, and hence the large radiative corrections to the lightest Higgs mass are necessary to satisfy the LEP bound. The large $\tan \beta$ implies small Higgsino masses and the lightest neutralino is Higgsino-rich. It tends to come around $m_{Z} / 2$ and an additional contribution to the invisible decay width of the $Z$-boson can be a problem. Nonetheless there are viable parameter sets.

There are numerous implications for collider phenomenology and cosmology. In the region $\tan \beta \lesssim 1$, the LHC is expected to discover supersymmetry through missing energy and dilepton signatures of squarks and gluinos. The trilepton signatures of charginos/neutralinos are viable at Tevatron and LHC unlike most anomaly-mediated models in the literature that make use of UV sensitive universal scalar mass. The lightest (SM-like) Higgs will decay mostly into $W W$ and $Z Z$ making it easy to find at the LHC. Finally, the abundance of the lightest supersymmetric particle, a neutralino that is mostly a singlino, could well be in the right range if its mass is of order $m_{Z} / 2$. The combination of LHC and LC would allow for a quantitative test of the predicted gaugino mass relation. In the other region $\tan \beta \gtrsim 5$, the squarks and gluinos may be beyond the reach of LHC. On the other hand, both wino-like and Higgsino-like charginos and neutralinos are light and can be looked for.

The model predicts that the gravitino is heavy, $\sim 100 \mathrm{TeV}$, solving its cosmological problem and allowing thermal leptogenesis to be a realistic possibility.

Finally, note that we have not attempted a full parameter scan of the model. We believe it would be useful to perform such a scan, combined with more detailed analysis of the precision electroweak constraint, the neutralino abundance, and the collider signatures. 


\section{Acknowledgments}

RK is the Marvin L. Goldberger Member and GDK is a Frank and Peggy Taplin Member; both of us thank these individuals for their generous support of the School of Natural Sciences. This work was supported by the Institute for Advanced Study, funds for Natural Sciences, as well as in part by the DOE under contracts DE-FG02-90ER40542 and DE-AC03-76SF00098 and in part by NSF grant PHY-0098840.

\section{References}

[1] R. Barbieri and G. F. Giudice, Nucl. Phys. B 306, 63 (1988); R. Barbieri and A. Strumia, arXiv:hep-ph/0007265.

[2] M. Dine and A. E. Nelson, Phys. Rev. D 48, 1277 (1993) [arXiv:hep-ph/9303230]; M. Dine, A. E. Nelson and Y. Shirman, Phys. Rev. D 51, 1362 (1995) [arXiv:hep-ph/9408384]; M. Dine, A. E. Nelson, Y. Nir and Y. Shirman, Phys. Rev. D 53, 2658 (1996) [arXiv:hep$\mathrm{ph} / 9507378]$.

[3] L. Randall and R. Sundrum, Nucl. Phys. B 557, 79 (1999) [arXiv:hep-th/9810155].

[4] G. F. Giudice, M. A. Luty, H. Murayama and R. Rattazzi, JHEP 9812, 027 (1998) [arXiv:hep-ph/9810442].

[5] D. E. Kaplan, G. D. Kribs and M. Schmaltz, Phys. Rev. D 62, 035010 (2000) [arXiv:hep$\mathrm{ph} / 9911293]$.

[6] Z. Chacko, M. A. Luty, A. E. Nelson and E. Ponton, JHEP 0001, 003 (2000) [arXiv:hep$\mathrm{ph} / 9911323]$.

[7] A. Pomarol and R. Rattazzi, JHEP 9905, 013 (1999) [arXiv:hep-ph/9903448].

[8] Z. Chacko, M. A. Luty, I. Maksymyk and E. Ponton, JHEP 0004, 001 (2000) [arXiv:hep$\mathrm{ph} / 9905390]$.

[9] E. Katz, Y. Shadmi and Y. Shirman, JHEP 9908, 015 (1999) [arXiv:hep-ph/9906296].

[10] J. A. Bagger, T. Moroi and E. Poppitz, JHEP 0004, 009 (2000) [arXiv:hep-th/9911029].

[11] I. Jack and D. R. T. Jones, Phys. Lett. B 482, 167 (2000) [arXiv:hep-ph/0003081].

[12] D. E. Kaplan and G. D. Kribs, JHEP 0009, 048 (2000) [arXiv:hep-ph/0009195].

[13] Z. Chacko and M. A. Luty, JHEP 0205, 047 (2002) [arXiv:hep-ph/0112172].

[14] N. Okada, Phys. Rev. D 65, 115009 (2002) [arXiv:hep-ph/0202219].

[15] A. E. Nelson and N. T. Weiner, arXiv:hep-ph/0210288.

[16] O. C. Anoka, K. S. Babu and I. Gogoladze, arXiv:hep-ph/0312176. 
[17] C. Csáki, J. Erlich, C. Grojean and G. D. Kribs, Phys. Rev. D 65, 015003 (2002) [arXiv:hep$\mathrm{ph} / 0106044]$.

[18] H. C. Cheng, D. E. Kaplan, M. Schmaltz and W. Skiba, Phys. Lett. B 515, 395 (2001) [arXiv:hep-ph/0106098].

[19] C. D. Froggatt and H. B. Nielsen, Nucl. Phys. B 147, 277 (1979).

[20] M. A. Luty and R. Sundrum, Phys. Rev. D 65, 066004 (2002) [arXiv:hep-th/0105137].

[21] M. Luty and R. Sundrum, Phys. Rev. D 67, 045007 (2003) [arXiv:hep-th/0111231].

[22] N. Arkani-Hamed, D. E. Kaplan, H. Murayama and Y. Nomura, JHEP 0102, 041 (2001) [arXiv:hep-ph/0012103].

[23] R. Harnik, H. Murayama and A. Pierce, JHEP 0208, 034 (2002) [arXiv:hep-ph/0204122].

[24] M. Carena, K. Huitu and T. Kobayashi, Nucl. Phys. B 592, 164 (2001) [arXiv:hepph/0003187].

[25] B. Murakami and J. D. Wells, Phys. Rev. D 68, 035006 (2003) [arXiv:hep-ph/0302209].

[26] R. Harnik, G. D. Kribs, D. T. Larson and H. Murayama, arXiv:hep-ph/0311349.

[27] E. Cremmer, B. Julia, J. Scherk, S. Ferrara, L. Girardello and P. van Nieuwenhuizen, Nucl. Phys. B 147, 105 (1979).

[28] E. Cremmer, S. Ferrara, L. Girardello and A. Van Proeyen, Nucl. Phys. B 212, 413 (1983).

[29] T. Kugo and S. Uehara, Nucl. Phys. B 226, 49 (1983); Nucl. Phys. B 222, 125 (1983).

[30] K. R. Dienes, C. F. Kolda and J. March-Russell, Nucl. Phys. B 492, 104 (1997) [arXiv:hep$\mathrm{ph} / 9610479]$.

[31] [LEP Collaboration], arXiv:hep-ex/0312023.

[32] See e.g. J. R. Ellis, J. F. Gunion, H. E. Haber, L. Roszkowski and F. Zwirner, Phys. Rev. D 39, 844 (1989).

[33] K. Agashe and M. Graesser, Nucl. Phys. B 507, 3 (1997) [arXiv:hep-ph/9704206].

[34] A. de Gouvêa, A. Friedland and H. Murayama, Phys. Rev. D 57, 5676 (1998) [arXiv:hep$\mathrm{ph} / 9711264]$.

[35] N. Seiberg, Phys. Rev. D 49, 6857 (1994) [arXiv:hep-th/9402044].

[36] See e.g. A. Kusenko, P. Langacker and G. Segre, Phys. Rev. D 54, 5824 (1996) [arXiv:hep$\mathrm{ph} / 9602414]$.

[37] Y. Okada, M. Yamaguchi and T. Yanagida, Prog. Theor. Phys. 85, 1 (1991).

[38] J. R. Ellis, G. Ridolfi and F. Zwirner, Phys. Lett. B 257, 83 (1991). 
[39] H. E. Haber and R. Hempfling, Phys. Rev. Lett. 66, 1815 (1991).

[40] I. Hinchliffe, F. E. Paige, M. D. Shapiro, J. Soderqvist and W. Yao, Phys. Rev. D 55, 5520 (1997) [arXiv:hep-ph/9610544].

[41] T. Tsukamoto, K. Fujii, H. Murayama, M. Yamaguchi and Y. Okada, Phys. Rev. D 51, 3153 (1995).

[42] G. D. Kribs, Phys. Rev. D 62, 015008 (2000) [arXiv:hep-ph/9909376].

[43] D. Cavalli et al., arXiv:hep-ph/0203056.

[44] T. Yanagida, in Proceedings of the Workshop on Unified Theory and Baryon Number of the Universe, eds. O. Sawada and A. Sugamoto (KEK, 1979) p.95; M. Gell-Mann, P. Ramond and R. Slansky, in Supergravity, eds. P. van Nieuwenhuizen and D. Freedman (North Holland, Amsterdam, 1979).

[45] T. Gherghetta, G. F. Giudice and J. D. Wells, Nucl. Phys. B 559, 27 (1999) [arXiv:hep$\mathrm{ph} / 9904378]$.

[46] T. Moroi and L. Randall, Nucl. Phys. B 570, 455 (2000) [arXiv:hep-ph/9906527].

[47] M. Kawasaki and T. Moroi, Prog. Theor. Phys. 93, 879 (1995) [arXiv:hep-ph/9403364].

[48] D. N. Spergel et al., Astrophys. J. Suppl. 148, 175 (2003) [arXiv:astro-ph/0302209].

[49] G. F. Giudice, A. Notari, M. Raidal, A. Riotto and A. Strumia, arXiv:hep-ph/0310123.

[50] W. Buchmüller, P. Di Bari and M. Plümacher, arXiv:hep-ph/0401240.

[51] G. D. Coughlan, R. Holman, P. Ramond and G. G. Ross, Phys. Lett. B 140, 44 (1984).

[52] B. de Carlos, J. A. Casas, F. Quevedo and E. Roulet, Phys. Lett. B 318, 447 (1993) [arXiv:hep-ph/9308325].

[53] T. Moroi, M. Yamaguchi and T. Yanagida, Phys. Lett. B 342, 105 (1995) [arXiv:hep$\mathrm{ph} / 9409367]$.

[54] M. Kawasaki, T. Moroi and T. Yanagida, Phys. Lett. B 370, 52 (1996) [arXiv:hep$\mathrm{ph} / 9509399]$.

[55] K. Rajagopal, M. S. Turner and F. Wilczek, Nucl. Phys. B 358, 447 (1991).

[56] T. Goto and M. Yamaguchi, Phys. Lett. B 276, 103 (1992).

[57] S. Kachru, M. B. Schulz and S. Trivedi, JHEP 0310, 007 (2003) [arXiv:hep-th/0201028].

[58] S. Gukov, S. Kachru, X. Liu and L. McAllister, arXiv:hep-th/0310159.

[59] T. Banks, D. B. Kaplan and A. E. Nelson, Phys. Rev. D 49, 779 (1994) [arXiv:hepph/9308292]. 
[60] I. Joichi and M. Yamaguchi, Phys. Lett. B 342, 111 (1995) [arXiv:hep-ph/9409266].

[61] P. B. Greene, K. Kadota and H. Murayama, Phys. Rev. D 68, 043502 (2003) [arXiv:hep$\mathrm{ph} / 0208276]$.

[62] J. Cline and S. Raby, Phys. Rev. D 43, 1781 (1991).

[63] J. A. Casas, J. R. Espinosa and I. Hidalgo, JHEP 0401, 008 (2004) [arXiv:hep-ph/0310137]. 